\title{
Insulin-like growth factor-1 improves postoperative cognitive dysfunction following splenectomy in aged rats
}

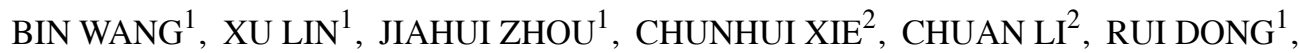 \\ GAOFENG ZHANG $^{1}$, XIAOPENG SUN ${ }^{1}$, MINGSHAN WANG $^{1 *}$ and YANLIN BI ${ }^{1 *}$ \\ ${ }^{1}$ Department of Anesthesiology, Qingdao Municipal Hospital Affiliated to Qingdao University, Qingdao, Shandong 266071; \\ ${ }^{2}$ Department of Anesthesiology, Weifang Medical University, Weifang, Shandong 261042, P.R. China
}

Received August 13, 2019; Accepted September 29, 2020

DOI: $10.3892 /$ etm. 2021.9647

\begin{abstract}
Postoperative cognitive dysfunction (POCD) is a serious complication following anesthesia and operations in aged patients undergoing surgical intervention. It is characterized by temporary or permanent cognitive decline, memory impairment and deterioration in language comprehension and social adaption ability. Therefore, the development of POCD prevention and treatment tools has become an area of interest. The current study assessed the therapeutic effects of insulin-like growth factor-1 (IGF-1) on POCD in aged rats and explored the underlying mechanisms. Model rats underwent splenectomy under $1.5-2 \%$ isoflurane and mechanical ventilation. IGF-1 $(50 \mu \mathrm{g} / \mathrm{kg})$ was diluted in normal saline and administered by abdominal hypodermic injection daily from the operation to day 7 post-operation. Following splenectomy, the animals showed marked cognitive impairment as determined by the Morris water maze test. Hippocampal protein levels of amyloid precursor protein (APP), $\beta$-site APP-cleaving enzyme-1 (BACE-1), amyloid- $\beta$ (A $\beta)$, capase 3 , Bax and Bcl-2 were assessed by immunoblotting. Neuronal apoptosis in the hippocampus was analyzed using a TUNEL assay. The results demonstrated that the levels of APP, BACE-1, A $\beta$, caspase 3 and Bax were increased following splenectomy, while the levels of $\mathrm{Bcl} 2$ were reduced at days 1, 3 and 7 post-operation in aged rats. However, IGF-1 downregulated APP, BACE-1, A $\beta$, capase 3 and $\mathrm{Bax}$, and upregulated $\mathrm{Bcl} 2$ at these time points following splenectomy. TUNEL staining revealed that administration of IGF-1 significantly reduced neuronal apoptosis in the hippocampal CA1 region following splenectomy. These
\end{abstract}

Correspondence to: Professor Yanlin $\mathrm{Bi}$, Department of Anesthesiology, Qingdao Municipal Hospital Affiliated to Qingdao University, 5 Donghai Middle Road, Qingdao, Shandong 266071, P.R. China

E-mail: yanlinbi68@sina.cn

${ }^{*}$ Contributed equally

Key words: postoperative cognitive dysfunction, insulin-like growth factor I, amyloid $\beta$, splenectomy, apoptosis, rat results indicated that IGF-1 decreased $\mathrm{A} \beta$-protein production and inhibited neuronal apoptosis in the hippocampus following splenectomy, subsequently alleviating POCD.

\section{Introduction}

Postoperative cognitive dysfunction (POCD) represents a serious complication following anesthesia and surgical procedures for patients undergoing surgical intervention (1). POCD is characterized by temporary or permanent cognitive decline, memory impairment, deterioration in language comprehension and social adaption ability, and particularly affects elderly people ( $>65$ years) (2). POCD can lead to increased mortality, prolonged hospitalization, other complications such as Alzheimer's disease and higher treatment costs (3). Although the pathogenic mechanisms for POCD remain unknown, its risk factors comprise trauma surgery, postoperative pain and neuronal apoptosis (4). Therefore, the development of POCD prevention and treatment tools has become a focus of interest for research.

Amyloid precursor protein (APP) is hydrolyzed in two ways: i) Degradation by $\alpha$-secretase during normal physiological conditions; or ii) generation of soluble $\beta$-APP8 and C99 by $\beta$-site APP-cleaving enzyme-1 (BACE-1), followed by C99 hydrolyzation by $\gamma$-secretase to generate insoluble amyloid- $\beta$ $(\mathrm{A} \beta)(5)$. APP is distributed in neuronal synapses (6). A $\beta$, a 36-43-amino acid peptide, is the main constituent of amyloid plaques in Alzheimer's disease (AD) (7). It is widely accepted that $A \beta$ oligomers are causally associated with the neurodegenerative processes accompanying $\mathrm{AD}(8)$. The most common isoforms of $A \beta$ are $A \beta 42$ and $A \beta 40$ (9), which serve important roles in POCD (10). A previous study revealed that POCD was associated with apoptosis of hippocampal neurons in rats (11). Therefore, effective inhibition of $\mathrm{A} \beta$ and apoptosis of hippocampal neurons demonstrated potential for the prevention and treatment of POCD.

Insulin-like growth factor I (IGF-I) serves critical roles in regulating body growth and metabolism and affects multiple cerebral functions (12). IGF-I promotes brain development, neuronal excitability, myelin sheath production, angiogenesis, synaptogenesis and neuronal survival, growth and differentiation (13). Additionally, IGF-I stimulates cell proliferation and survival in multiple cell types (14-16) and is considered a 
universal cytoprotective molecule, protecting cells from free radicals and apoptosis (17). Notably, a reduction in the amount of IGF-1 markedly contributed to age-associated cognitive impairment (18). A previous study revealed that IGF-1 expression was negatively associated with the progression of cognitive impairment (19).

Therefore, the current study aimed to assess whether IGF-I improved POCD by mediating apoptosis and $\mathrm{A} \beta$ production. The present study studied cognitive function in aged rats following surgery with or without IGF-I administration to investigate the protective effects of IGF-I on splenectomyinduced POCD.

\section{Materials and methods}

Animals and groups. A total of 150 male Wistar rats (age, 16-18 months; weight, 350-550 g) were purchased from the SPF Beijing Biotechnology Co., Ltd. (license no. SCXK2016-0002) and examined. The animals were housed under a 12-hour light/dark cycle with free access to water and rodent chow. All animal experiments followed the Guidance Suggestions for the Care and Use of Laboratory Animals by the Ministry of Science and Technology of the People's Republic of China (20). Approval was obtained from the Animal Ethics Committee of Qingdao Municipal Hospital (Qingdao, China). Rats were housed under standard conditions with food and water available ad libitum and were allowed to acclimatize at 24-26 ${ }^{\circ} \mathrm{C}$ for 1 week prior to experiments. The living environment of the rats was clean and tidy and suitable for survival. The rats were randomized into five groups ( $\mathrm{n}=30 /$ group), as follows: i) Control (C); ii) isoflurane (I); iii) splenectomy (S); iv) $\mathrm{S}+$ normal saline $(\mathrm{S}+\mathrm{NS})$ and v) $\mathrm{S}+\mathrm{IGF}-1$ ( $\mathrm{S}+\mathrm{IGF}-1)$.

Surgery and injection. The control group underwent no treatment. Rats in the I group were given continuous inhalation of $1.5-2 \%$ isoflurane for intubation and given $1.5 \%$ isoflurane and mechanical ventilation with $100 \%$ oxygen for anesthesia maintenance. This anesthetic procedure was selected due to its clinical relevance; additionally, anesthetics are considered to play a role in cognitive impairment (21). Rats in Group $S$ underwent splenectomy following the same anesthesia as those in Group I. Briefly, the animals were placed in a supine position followed by skin shaving. After disinfection, an incision was made $1.5-2.0 \mathrm{~cm}$ below the costal margin for spleen removal. The splenic artery and vein were ligatured with silk threads. The abdominal cavity was closed after hemostasis and bupivacaine $(0.25 \%)$ was administered subcutaneously prior to wound closure to ensure that the rats did not undergo pain during and following the operation (22). IGF-1 $(50 \mu \mathrm{g} / \mathrm{kg}$; cat. no. 50437-MNAY; Sino Biological) was diluted in NS and administered via abdominal hypodermic injection every day from splenectomy to 7 days post-surgery in the $S+$ I GF-1 group. In the S + NS group, equal volumes of NS were administered via abdominal hypodermic injection every day from splenectomy to 7 days post-surgery.

Current POCD models are achieved by splenectomy (23), partial hepatectomy (24) and limb orthopedic surgery (25). Splenectomy results in postsurgical reversible learning and memory dysfunction, subsequently inducing POCD (26). Furthermore, splenectomy shares significant similarity with clinical abdominal surgery (short surgery time, controllability, high success rate and reduced mortality) (27). Therefore, splenectomy is often performed to induce a model of POCD (28).

After the rats were anesthetized with $4-5 \%$ isoflurane for 5-7 min, reflexes disappeared. When the monitor indicated cardiac arrest, respiratory arrest and pupil dilation, the rats were euthanized by cervical dislocation.

Morris Water Maze (MWM). To assess learning and memory abilities, MWM tests were performed as described previously (29). This assay can identify animals presenting with sensorimotor dysfunction and was used to assess cognitive function.

The MWM system (Shanghai XinRuan Information Technology Co., Ltd.) comprised an imaging device for swimming tracking and a circular test pool (diameter, $120 \mathrm{~cm}$; height, $40 \mathrm{~cm}$ ) with a cylindrical platform (diameter, $10 \mathrm{~cm}$; height, $30 \mathrm{~cm}) 2 \mathrm{~cm}$ below the water surface. For the swimming test, animals were allowed to adapt to the platform for $30 \mathrm{sec}$ before entering the water from four different quadrants on the pool wall. The time spent before finding the platform (escape latency) was recorded to assess learning and memory abilities prior to surgery. Animals that could not find the platform were guided within $60 \mathrm{sec}$ to it and allowed a $10-\mathrm{sec}$ adaptation. The swimming test was performed 4 trials/day for 5 consecutive days starting from 1 week prior to surgery. In the spatial test, the platform was removed prior to animal placement in the water for $60 \mathrm{sec}$ and the ratio of swimming time in the target quadrant was evaluated. The spatial test was performed on the day preceding the operation and at postoperative days 1, 3 and 7. Escape latency and swimming distance in the target quadrant were analyzed.

Western blotting. Western blotting was performed as previously reported (30) to detect proteins. The hippocampus was lysed in RIPA lysis buffer (Beyotime Institute of Biotechnology) and protease inhibitors (PMSF; Beyotime Instittue of Biotechnology), homogenized and placed in ice for full lysis for $40 \mathrm{~min}$, followed by $20 \mathrm{~min}$ of centrifugation $\left(1,600 \times \mathrm{g} ; 4^{\circ} \mathrm{C}\right)$. Total protein was quantified using a bicinchoninic acid assay kit. Proteins (30 $\mu \mathrm{g} /$ lane) were resolved by $10 \%$ SDS-PAGE and electrotransferred onto polyvinylidene fluoride membranes (EMD Millipore), which were blocked with $10 \%$ skimmed milk containing TBS-Tween-20 (TBS-T; $0.1 \%$ ) in ambient conditions for $1 \mathrm{~h}$ at room temperature. This was followed by overnight incubation at $4^{\circ} \mathrm{C}$ with the following primary antibodies: Anti- $\beta$-actin (1:2,000; cat. no. ab8226; Abcam), anti-APP (1:2,000; cat. no. ab32136; Abcam), antiBACE1 (1:1,000; cat. no. ab183612; Abcam), anti-caspase-3 (1:200; cat. no. ab13847; Abcam), anti-Bax (1:200; cat. no. ab32503; Abcam), anti-Bcl2 (1:200; cat. no. ab32124; Abcam) and anti-A $\beta$ (1:2,000; cat. no. ab126649; Abcam). Following overnight incubation, the membranes were washed with TBS-T three times (10 min each) and were further incubated with goat anti-mouse secondary antibody (1:5,000; cat. no. SAA544Mu19; Cloud-Clone Corp.) and goat anti-rabbit secondary antibody (1:5,000; cat. no. bs-0295G-HRP; Bioss) for $1 \mathrm{~h}$ at room temperature. membranes were then washed with TBS-T three times (10 min each). The generated immune complexes were detected with an enhanced chemiluminescence detection system (Amersham; Cytiva), and visualization 
A

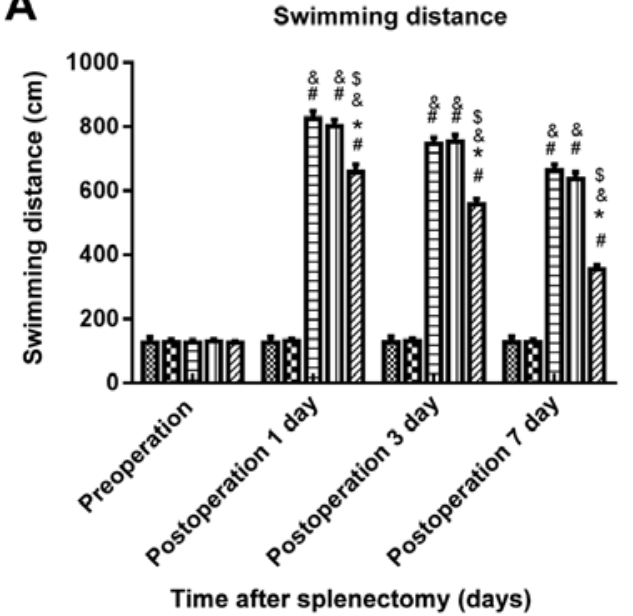

B

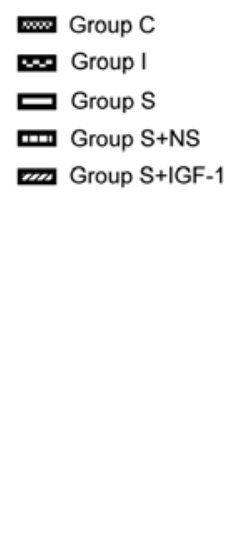

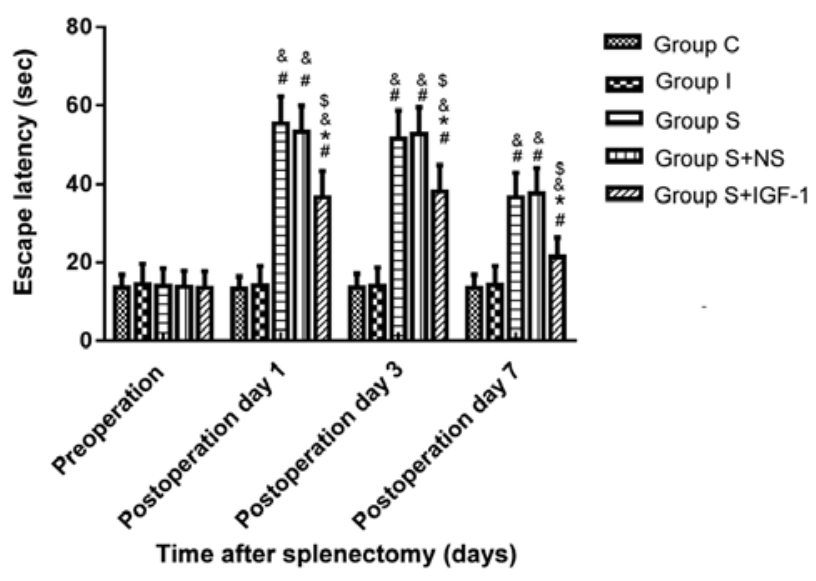

Figure 1. Neurocognitive tests were performed using the Morris water maze. The results revealed that (A) Swimming distance and (B) Escape latency increased in the $\mathrm{S}, \mathrm{S}+\mathrm{NS}$ and $\mathrm{S}+\mathrm{IGF}-1$ groups compared with the $\mathrm{C}$ and I groups at days 1,3 and 7 post-surgery. ${ }^{\#} \mathrm{P}<0.05$ vs. $\mathrm{C}$ and ${ }^{\text {\& }} \mathrm{P}<0.05$ vs. I. ${ }^{\text {P }}<0.05$ vs. S and ${ }^{\mathrm{S}} \mathrm{P}<0.05$ vs. S + NS. C, control; I, isoflurane; S, splenectomy; NS, normal saline; IGF-1, insulin-like growth factor-1.

A

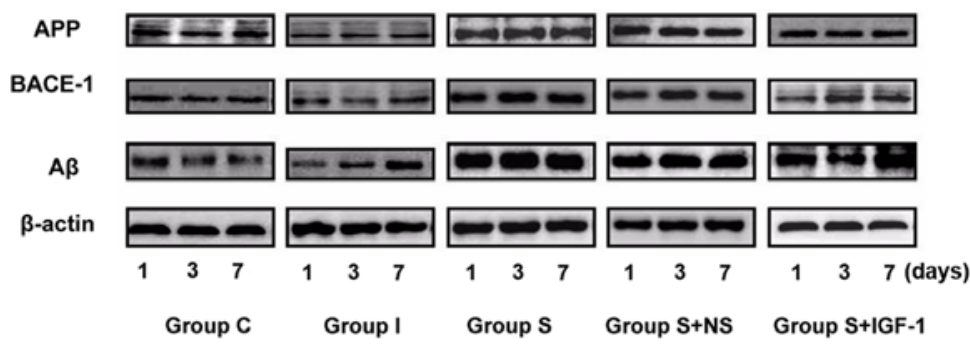

C

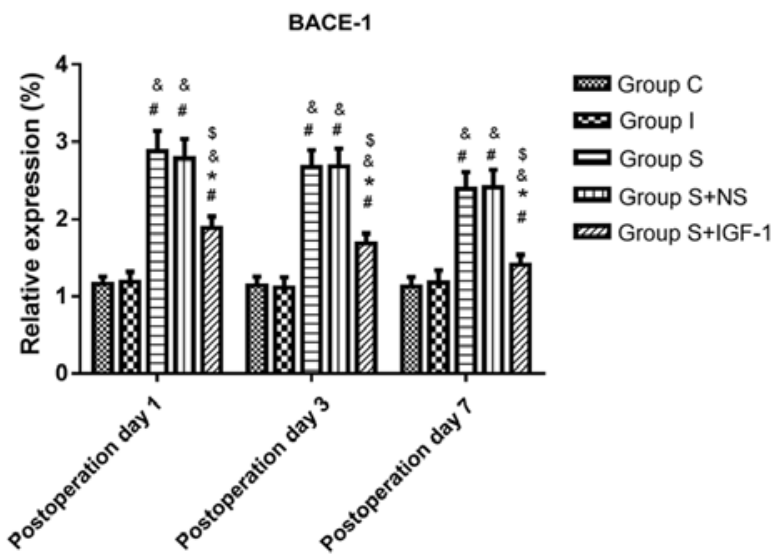

Time after splenectomy (days)
B

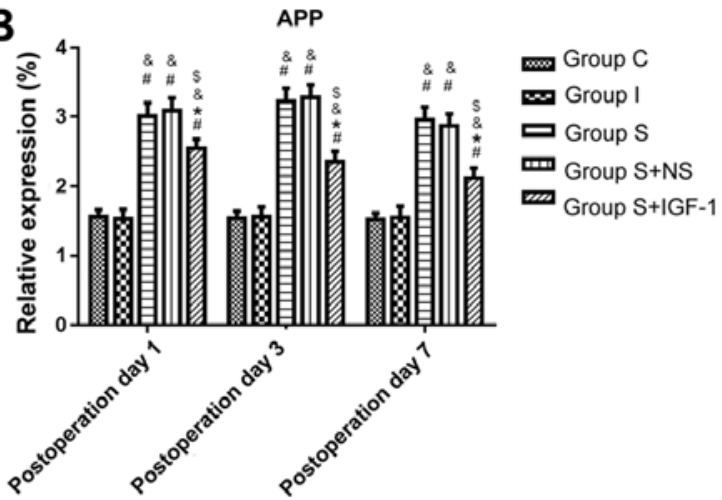

Time after splenectomy (days)

D $A \beta$
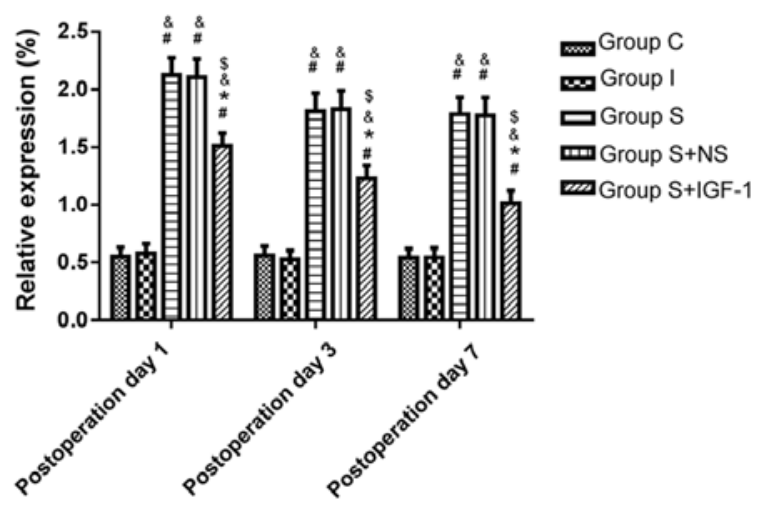

Time after splenectomy (days)

Figure 2. Hippocampal levels of APP, BACE-1 and A $\beta$ after splenectomy at different timepoints. (A) IGF reduces the levels of (B) APP, (C) BACE-1 and (D) A $\beta$ in the hippocampus of aged rats at days 1,3 and 7 post-surgery. ${ }^{"} \mathrm{P}<0.05$ vs. $\mathrm{C}$ and ${ }^{\circledR} \mathrm{P}<0.05$ vs. I. ${ }^{*} \mathrm{P}<0.05$ vs. $\mathrm{S}$ and ${ }^{\$} \mathrm{P}<0.05$ vs. $\mathrm{S}+\mathrm{NS}$. IGF-1, insulin-like growth factor-1; APP, amyloid precursor protein; BACE-1, $\beta$-site APP-cleaving enzyme-1; A $\beta$, amyloid- $\beta$; $C$, control; I, isoflurane; S, splenectomy; NS, normal saline.

was performed with the Sygene Bio Image system (Vilber). Gray values for APP, BACE-1, A 3 , caspase3, Bax and Bcl-2 were detected by ImageJ 1.8.0 (National Institutes of Health). The ratio of the target protein to the internal control, $\beta$-actin, was used in the final analysis.
TUNEL assay. TUNEL staining was performed according to a previous study (31). Neuronal apoptosis in hippocampal samples was analyzed by TUNEL assays. The samples were fixed with $4 \%$ paraformaldehyde at $4^{\circ} \mathrm{C}$ for $4 \mathrm{~h}$. Following this, samples were treated with $3 \%$ hydrogen peroxidase 
A

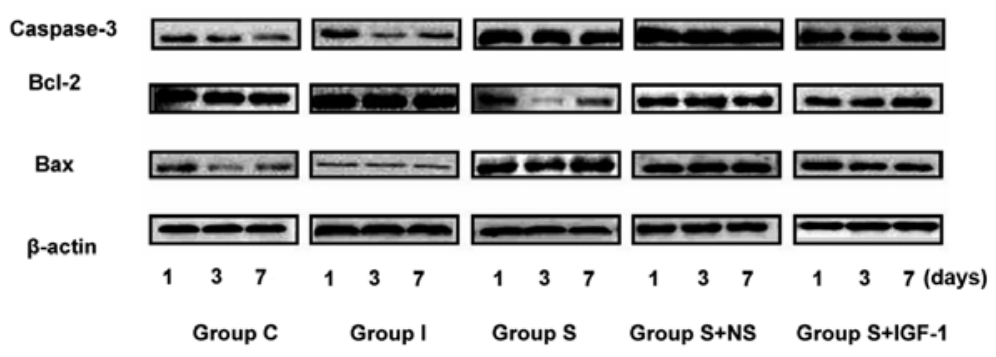

C

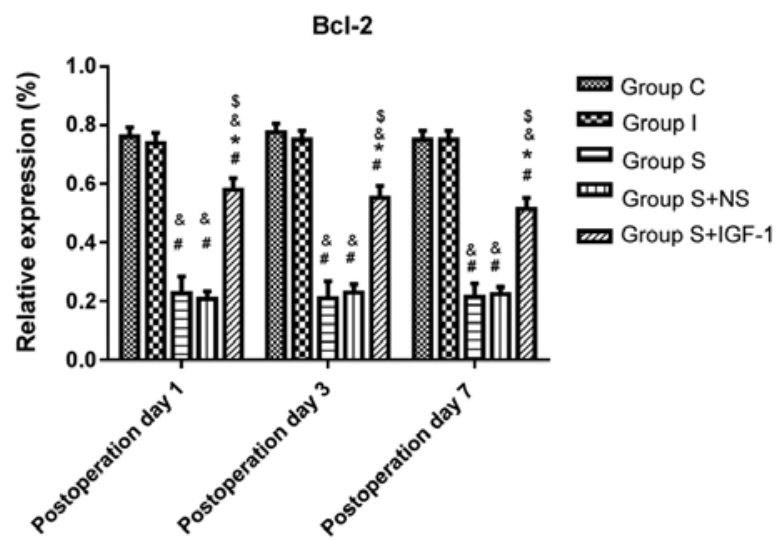

Time after splenectomy (days)

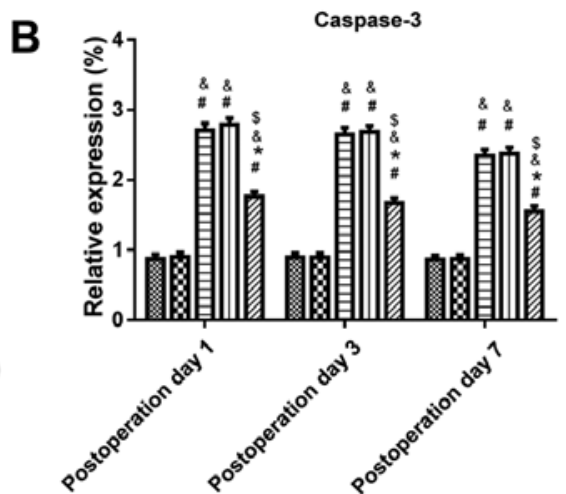

Time after splenectomy (days)

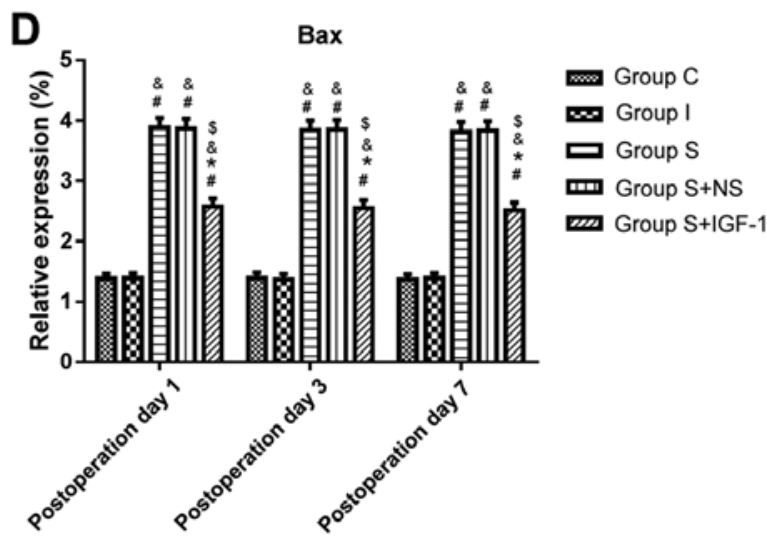

Time after splenectomy (days)

Figure 3. Hippocampal levels of caspase-3, Bax and Bcl-2 after splenectomy at different timepoints. (A) IGF reduces the expression amounts of (B) caspase-3, (C) Bax and (D) Bcl-2 in the hippocampal samples of aged rats at days 1,3 and 7 post-surgery. ${ }^{~} \mathrm{P}<0.05$ vs. $\mathrm{C}$ and ${ }^{\circledR} \mathrm{P}<0.05$ vs. I. ${ }^{*} \mathrm{P}<0.05$ vs. $\mathrm{S}$ and ${ }^{\$} \mathrm{P}<0.05$ vs. S + NS. IGF-1, insulin-like growth factor-1; C, control; I, isoflurane; S, splenectomy; NS, normal saline.

and incubated in a labeling reaction mixture comprised of terminal deoxynucleotidyl transferase and deoxynucleotides overnight at $4^{\circ} \mathrm{C}$. Sections were then subjected to further incubation with horseradish peroxidase (1:500; Shanghai Macklin Biochemical Co., Ltd.) for $30 \mathrm{~min}$ and treatment with 3,3'-diaminobenzidine for $15 \mathrm{~min}$ at $37^{\circ} \mathrm{C}$ in the dark. Reactions were stopped with running water and counterstaining was performed with hematoxylin at $37^{\circ} \mathrm{C}$ for $10 \mathrm{~min}$. Following dehydration with a graded ethyl alcohol series and xylene treatment, tissue samples were mounted on coverslips with neutral gum. Apoptotic nuclei appeared as dark brown dots. Apoptotic cells in the CA1 region were assessed by light microscopy (magnification, $\mathrm{x} 400$ ) in a blinded manner in five random high-power fields.

Statistical analysis. Data are presented as the mean \pm SD. All parameters were assessed by one-way ANOVA followed by Tukey's post hoc test. SPSS (version no. 20.0; IBM Corp.) was used for data analysis. $\mathrm{P}<0.05$ was considered to indicate a statistically significant difference.

\section{Results}

Cognitive function declines in aged rats following splenectomy. The MWM was performed to assess spatial learning and memory abilities. In Group S, the swimming distance (Fig. 1A) and escape latency (Fig. 1B) were significantly longer at days 1, 3 and 7 post-surgery compared with the $\mathrm{C}$ and I groups. The $\mathrm{C}$ and I groups presented similar values for swimming distance and escape latency throughout the experiment. These results indicated that surgery aggravated cognitive impairment. Swimming distance and escape latency in the S + IGF-1 group were significantly shorter at days 1, 3 and 7 post-surgery compared with the S and S + NS groups, indicating that IGF-1 improved cognitive function following splenectomy.

IGF- 1 decreases $A \beta$ protein production in the hippocampus of aged rats following splenectomy. Protein levels of APP, BACE-1 and A $\beta$ were assessed in hippocampal specimens following surgery by immunoblotting. Splenectomy significantly upregulated APP, BACE- 1 and A $\beta$ expression at the protein level in hippocampal samples from aged rats at days 1, 3 and 7 compared with the $\mathrm{C}$ and I groups (Fig. 2). IGF-1 administration following splenectomy significantly decreased the protein amounts of APP, BACE- 1 and A $\beta$ in the hippocampus of aged rats at days 1, 3 and 7 compared with the $\mathrm{S}+\mathrm{NS}$ group.

IGF-1 inhibits the apoptosis of neurons in the hippocampal CAl region in aged rats following splenectomy. Immunoblotting was performed to assess the protein levels of capase-3, Bax and $\mathrm{Bcl} 2$ in rat hippocampi following surgery. 


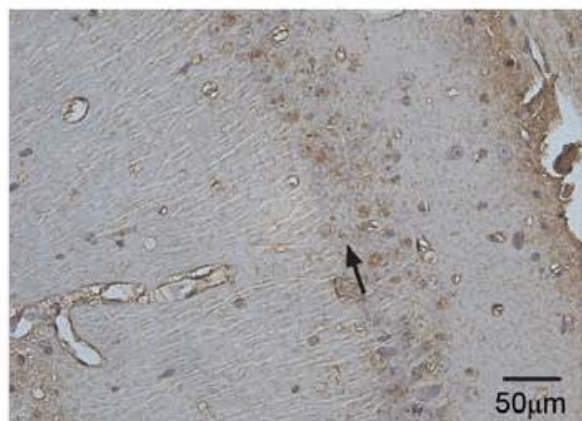

Group C

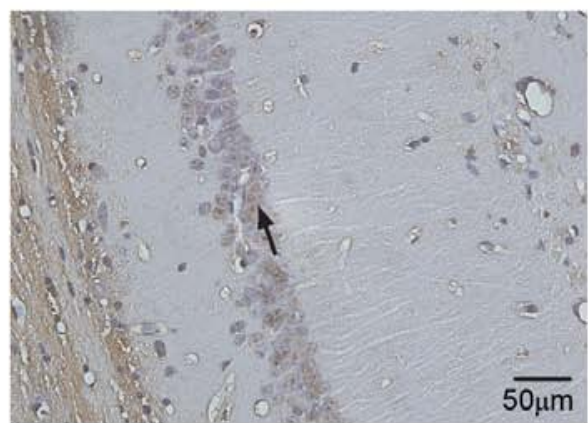

Group I

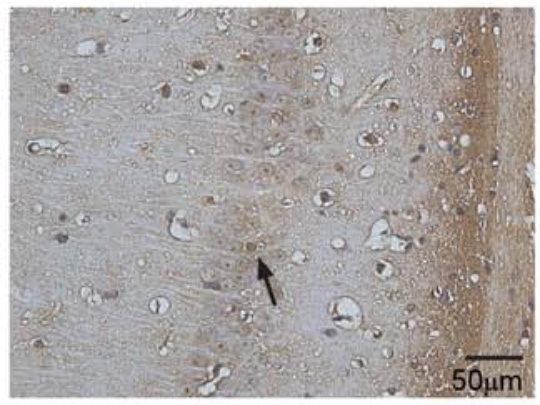

Group S

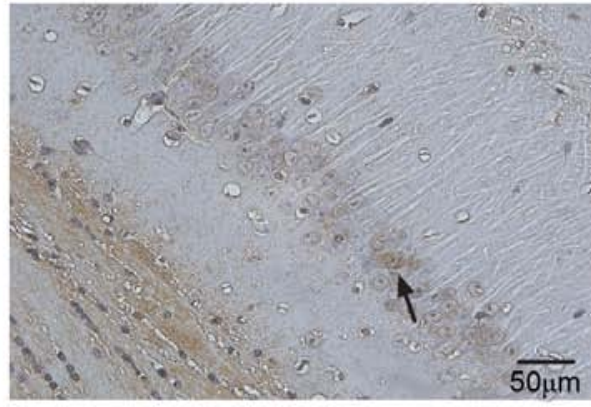

Group S+NS

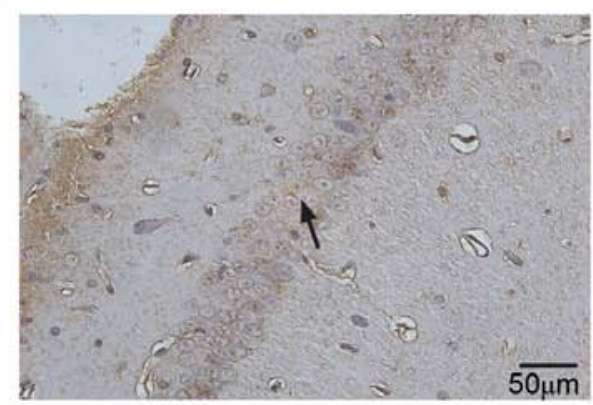

Group S+IGF-1

Figure 4. TUNEL staining results of the hippocampal CA1 region of rats. The numbers of neurons were reduced in the C and I groups. Markedly increased numbers of apoptotic neurons and neuron shrinking were observed in S and S + NS groups. The number of apoptotic neurons markedly decreased in the $\mathrm{S}+\mathrm{IGF}-1$ group compared with the S and S + NS groups. Black arrows indicate apoptotic neurons. Scale bar, $50 \mu \mathrm{m}$. C, control; I, isoflurane; S, splenectomy; NS, normal saline; IGF-1, insulin-like growth factor-1.

The results demonstrated that splenectomy significantly upregulated caspase- 3 and Bax expression, and significantly downregulated $\mathrm{Bcl} 2$ expression in the hippocampal CA1 region of aged rats at days 1,3 and 7 post-surgery compared with the $\mathrm{C}$ and I groups (Fig. 3). Furthermore, IGF-1 administration following splenectomy significantly reduced capase-3 and Bax protein levels, and significantly increased Bcl2 levels in hippocampal samples in aged rats at days 1,3 and 7 postsurgery compared with the S and S + NS groups.

The TUNEL assay revealed that IGF-1 administration markedly reduced neuronal apoptosis associated with surgery in the hippocampal CA1 region of animals in the S + IGF-1 group compared with the S and S + NS groups (Fig. 4).

\section{Discussion}

The current study demonstrated that swimming distance and escape latency increased post-operatively, indicating splenectomy induced POCD in aged rats. This was consistent with previous studies which indicated markedly aggravated cognitive dysfunction in rats that underwent splenectomy $(27,32)$. Furthermore, the results of the current study revealed that splenectomy induced the overexpression of APP, BACE-1 and $A \beta$ in the hippocampus. This indicated that changes in $A \beta$-protein may be associated with early POCD, which is consistent with findings published by Canet et al (33). Furthermore, IGF-1, a multifunctional polypeptide essential for normal growth and development (12), inhibited the production of upstream proteins APP and BACE-1, attenuated A $\beta$ production and improved surgery-induced POCD. These results indicated IGF-1 had a protective role in POCD by attenuating $A \beta$ production in aged rats. Cognitive dysfunction persists transiently due to the acute production of APP and $\mathrm{A} \beta$ (34). Neuroinflammation associated with $\mathrm{A} \beta$ aggregation is an essential factor in cognitive dysfunction (35). Cleavage of APP by BACE- 1 produced soluble $\beta$-APP8 and C99, and C99 is hydrolyzed by $\gamma$-secretase to produce insoluble $A \beta$ (36). Research has demonstrated that $A \beta$ is located in the brain as metastable monomeric A $\beta$ is constantly produced by APP under conditions of catalysis by secretases (37). AD and POCD have similar neuropathogenesis (38). Given that cognitive function is unavoidably impaired by major surgeries in aged patients, developing efficient therapeutic tools is of high significance (39). The present work recommended IGF-1 as a novel potent drug as it improved POCD by reducing the generation of A $\beta$. A previous study reported that IGF-1 inhibited JNK activity (40) and enhanced APP phosphorylation at Thr668 in rat hippocampal tissue (41). Furthermore, IGF-1 increased $\alpha$-secretase expression in the hippocampus and lowered the levels of BACE1 and $\gamma$-secretase, thereby reducing the levels and deposition of $\mathrm{A} \beta$ in hippocampal tissue (42).

Additionally, the results of the current study revealed that IGF-1 downregulated caspase-3 and Bax, and upregulated $\mathrm{Bcl} 2$ following splenectomy in hippocampal samples at days 1 , 3 and 7 post-surgery. IGF-1 administration markedly reduced neuronal apoptosis associated with surgery in the hippocampal CA1 region of rats. Apoptosis is another important mechanism for POCD development (43). The present study demonstrated that in the hippocampus of aged rats following splenectomy, caspase-3 and Bax were significantly increased, 
while Bcl-2 was significantly decreased. The Bcl-2 family protein is located upstream of the mitochondria and is an important regulator of mitochondrial membrane permeability, which controls the release of cytochrome $\mathrm{c}$ and activates downstream caspase- 3 proteases, mediating cell survival or death $(44,45)$. Under the condition of apoptosis inducer signals, caspases are activated by the combination of specific cofactors (46). Once caspases are activated, degradation of cellular proteins occurs, eventually causing irreversible cell death (47). Bcl-2 localization in the outer membrane of mitochondria is mediated by the indirect action of the caspases (48). Apoptotic protease activating factor 1 is targeted to the mitochondrial membrane by Bcl-2, which blocks the activation of the apoptotic protease by regulating its structure and regulates the action of cry-e (49). IGF-1 prevents apoptosis by inducing the signaling pathway mediated by PI3K and its downstream target Akt (50). The interaction between IGF-1 and the IGF-1 receptor phosphorylates tyrosine kinase or activates PI3K by activating the insulin receptor substrate (51). When activated, PI3K subunits phosphorylate phosphoinositide-dependent protein kinases and activate gene expression and protein translation of downstream target Akt. When the upstream signal activates the main target enzyme Akt, anti-apoptotic genes are upregulated, and Akt regulates Bcl-2 protein expression and enhances Bcl-2 activity (52). Peruzzi et al (53) demonstrated that PI3K inhibitors prevented IGF-1 from upregulating Bax and downregulating Bcl-2. Therefore, IGF-1 inhibited the apoptotic pathway (54).

Certain limitations of the current study must be discussed. Firstly, the number of animal experiments was limited. Sample sizes will be increased in future experiments. Secondly, the behavioral memory of rats can be measured comprehensively using behavior tests, including contextual fear conditioning test and the elevated plus maze test. Thirdly, since animal models cannot completely reproduce complex clinical situations, it remains essential to confirm whether similar changes occur in patients following surgery.

Overall, the current study reported a neuroprotective role for IGF-1 for POCD in aged rats. The mechanism involved decreased $A \beta$-protein production and inhibited neuronal apoptosis in the hippocampus. The present results indicated the use of IGF-1 for preventing POCD in aged patients.

\section{Acknowledgements}

Not applicable.

\section{Funding}

The current study was funded by the Qingdao Medical Research Guidance Program, Qingdao, China (grant no. 2018-WJZD011),

\section{Availability of data and materials}

The datasets used and/or analyzed during the current study are available from the corresponding author on reasonable request.

\section{Authors' contributions}

YB and MW conceived and designed the current study and drafted the manuscript. CX, CL and JZ performed the experiments at the physical laboratory of Qingdao University, China. $\mathrm{RD}, \mathrm{XL}$ and XS analyzed data. GZ and BW performed the experiments and wrote and revised the manuscript. All authors read and approved the final manuscript.

\section{Ethics and approval and consent to participate}

The present study followed the recommendations of the National Institute of Health guidelines for the care and use of laboratory animals and obtained approval from the Clinical Trial Ethics Committee of Qingdao Municipal Hospital, Qingdao, China.

\section{Patient consent for publication}

Not applicable.

\section{Competing interests}

The authors declare that they have no competing interests.

\section{References}

1. Safavynia SA and Goldstein PA: The role of neuroinflammation in postoperative cognitive dysfunction: Moving from hypothesis to treatment. Front Psychiatry 9: 752, 2019.

2. Ramaiah R and Lam AM: Postoperative cognitive dysfunction in the elderly. Anesthesiol Clin 27: 485-496, 2009.

3. Steinmetz J, Christensen KB, Lund T, Lohse N and Rasmussen LS; ISPOCD Group: Long-term consequences of postoperative cognitive dysfunction. Anesthesiology 110: 548-555, 2009.

4. Wuri G, Wang DX, Zhou Y and Zhu SN: Effects of surgical stress on long-term memory function in mice of different ages. Acta Anaesthesiol Scand 55: 474-485, 2011.

5. Songjiang Z and Lixiang W: Amyloid-beta associated with chitosan nano-carrier has favorable immunogenicity and permeates the BBB. AAPS PharmSciTech 10: 900-905, 2009.

6. Hoe HS, Lee HK and Pak DT: The upside of APP at synapses. CNS Neurosci Ther 18: 47-56, 2012.

7. Poulsen SA, Watson AA, Fairlie DP and Craik DJ: Solution structures in aqueous SDS micelles of two amyloid beta peptides of A beta(1-28) mutated at the alpha-secretase cleavage site (K16E, K16F). J Struct Biol 130: 142-152, 2000.

8. Garcez ML, Mina F, Bellettini-Santos T, da Luz AP, Schiavo GL, Macieski JMC, Medeiros EB, Marques AO, Magnus NQ and Budni J: The involvement of NLRP3 on the effects of minocycline in an AD-like pathology induced by $\beta$-amyloid oligomers administered to mice. Mol Neurobiol 56: 2606-2617, 2019.

9. Ghahghaei A, Bathaie SZ, Kheirkhah H and Bahraminejad E: The protective effect of crocin on the amyloid fibril formation of A 342 peptide in vitro. Cell Mol Biol Lett 18: 328-339, 2013.

10. Wu Z, Zhang M, Zhang Z, Dong W, Wang Q and Ren J: Ratio of $\beta$-amyloid protein $(A \beta)$ and Tau predicts the postoperative cognitive dysfunction on patients undergoing total hip/knee replacement surgery. Exp Ther Med 15: 878-884, 2018.

11. Zhang X, Dong H, Li N, Zhang S, Sun J, Zhang S and Qian Y: Activated brain mast cells contribute to postoperative cognitive dysfunction by evoking microglia activation and neuronal apoptosis. J Neuroinflammation 13: 127, 2016.

12. Vassilakos G and Barton ER: Insulin-like growth factor I regulation and its actions in skeletal muscle. Compr Physiol 9: 413-438, 2018.

13. D'Ercole AJ, Ye P and O'Kusky JR: Mutant mouse models of insulin-like growth factor actions in the central nervous system. Neuropeptides 36: 209-220, 2002. 
14. Mishra N, Lata S, Deshmukh P, Kamat K, Surolia A and Banerjee T: Insulin signaling pathway protects neuronal cell lines by Sirt 3 mediated IRS2 activation. Biofactors 44: 224-236, 2018.

15. Liao R, Yan F, Zeng Z, Farhan M, Little P, Quirion R, Srivastava LK and Zheng W: Amiodarone-Induced Retinal Neuronal Cell Apoptosis Attenuated by IGF-1 via Counter Regulation of the PI3k/Akt/FoxO3a Pathway. Mol Neurobiol 54 6931-6943, 2017.

16. Nakao Y, Otani H, Yamamura T, Hattori R, Osako M and Imamura $\mathrm{H}$ : Insulin-like growth factor 1 prevents neuronal cell death and paraplegia in the rabbit model of spinal cord ischemia J Thorac Cardiovasc Surg 122: 136-143, 2001.

17. Lackey BR, Gray SL and Henricks DM: Actions and interactions of the IGF system in Alzheimer's disease: Review and hypotheses. Growth Horm IGF Res 10: 1-13, 2000.

18. Sonntag WE1: Deak F, Ashpole N, Toth P, Csiszar A, Freeman W, Ungvari Z: Insulin-like growth factor-1 in CNS and cerebrovascular aging. Front Aging Neurosci 5: 27, 2013.

19. Angelini A, Bendini C, Neviani F, Bergamini L, Manni B, Trenti T, Rovati R and Neri M: Insulin-like growth factor-1 (IGF-1): Relation with cognitive functioning and neuroimaging marker of brain damage in a sample of hypertensive elderly subjects. Arch Gerontol Geriatr 49 (Suppl 1): 5-12, 2009.

20. Guidance Suggestions for the Care and Use of Laboratory Animals 9: 30, 2006

21. Umholtz M and Nader ND: Anesthetic immunomodulation of the neuroinflammation in postoperative cognitive dysfunction. Immunol Invest 46: 805-815, 2017.

22. Newman S, Stygall J, Hirani S, Shaefi S, Maze M and Warltier DC: Postoperative cognitive dysfunction after noncardiac surgery: A systematic review. Anesthesiology 106: 572-590, 2007.

23. Wan Y, Xu J, Ma D, Zeng Y, Cibelli M and Maze M: Postoperative impairment of cognitive function in rats: A possible role for cytokine-mediated inflammation in the hippocampus. Anesthesiology 106: 436-443, 2007.

24. Li M, Yong-Zhe L, Ya-Qun M, Sheng-Suo Z, Li-Tao Z and Ning-Ling P: Ulinastatin alleviates neuroinflammation but fails to improve cognitive function in aged rats following partial hepatectomy. Neurochem Res 38: 1070-1077, 2013.

25. Cibelli M,Fidalgo AR, Terrando N,MaD,Monaco C,Feldmann M Takata M, Lever IJ, Nanchahal J, Fanselow MS, et al: Role of interleukin-1beta in postoperative cognitive dysfunction. Ann Neurol 68: 360-368, 2010

26. Wang B, Li S, Cao X, Dou X, Li J, Wang L, Wang M and Bi Y: Blood-brain barrier disruption leads to postoperative cognitive dysfunction. Curr Neurovasc Res 14: 359-367, 2017.

27. Yu L, Sun L and Chen S: Protective effect of senegenin on splenectomy-induced postoperative cognitive dysfunction in elderly rats. Exp Ther Med 7: 821-826, 2014.

28. Kamer AR, Galoyan SM, Haile M, Kline R, Boutajangout A Li YS and Bekker A: Meloxicam improves object recognition memory and modulates glial activation after splenectomy in mice. Eur J Anaesthesiol 29: 332-337, 2012.

29. Weitzner DS, Engler-Chiurazzi EB, Kotilinek LA, Ashe KH and Reed MN: Morris water maze test: Optimization for mouse strain and testing environment. J Vis Exp 22: e52706, 2015.

30. Jawa RS, Anillo S, Huntoon K, Baumann H and Kulaylat M: Interleukin-6 in surgery, trauma, and critical care part II: Clinical implications. J Intensive Care Med 26: 73-87, 2011.

31. Pang H, Huang T, Song J, Li D, Zhao Y and Ma X: Inhibiting HMGB1 with glycyrrhizic acid protects brain injury after DAI via its anti-inflammatory effect. Mediators Inflamm 2016: 4569521,2016

32. Lu SM, Gui B, Dong HQ, Zhang X, Zhang SS, Hu LQ, Liu HL, Sun J and Qian YN: Prophylactic lithium alleviates splenectomy-induced cognitive dysfunction possibly by inhibiting hippocampal TLR4 activation in aged rats. Brain Res Bull 114 31-41, 2015.

33. Canet J, Raeder J, Rasmussen LS, Enlund M, Kuipers HM, Hanning CD, Jolles J, Korttila K, Siersma VD, Dodds C, et al; ISPOCD2 investigators: Cognitive dysfunction after minor surgery in the elderly. Acta Anaesthesiol Scand 47: 1204-1210, 2003.

34. Roher AE, Kokjohn TA, Clarke SG, Sierks MR, Maarouf CL, Serrano GE, Sabbagh MS and Beach TG: APP/A $\beta$ structural diversity and Alzheimer's disease pathogenesis. Neurochem Int 110: $1-13,2017$
35. Zhu D, Yang N, Liu YY, Zheng J, Ji C and Zuo PP: M2 Macrophage transplantation ameliorates cognitive dysfunction in amyloid- $\beta$-treated rats through regulation of microglial polarization. J Alzheimers Dis 52: 483-495, 2016.

36. Masters CL and Selkoe DJ: Biochemistry of amyloid $\beta$-protein and amyloid deposits in Alzheimer disease. Cold Spring Harb Perspect Med 2: a006262, 2012.

37. Kulic L, McAfoose J, Welt T, Tackenberg C, Späni C, Wirth F, Finder V, Konietzko U, Giese M, Eckert A, et al: Early accumulation of intracellular fibrillar oligomers and late congophilic amyloid angiopathy in mice expressing the Osaka intra-A $\beta$ APP mutation. Transl Psychiatry 2: e183, 2012.

38. Xie Z and Tanzi RE; XieZ: Alzheimer's disease and postoperative cognitive dysfunction. Exp Gerontol 41: 346-359, 2006.

39. Qian XL, Zhang W, Liu MZ, Zhou YB, Zhang JM, Han L, Peng YM, Jiang JH and Wang QD: Dexmedetomidine improves early postoperative cognitive dysfunction in aged mice. Eur J Pharmacol 746: 206-212, 2015

40. Teng JA, Wu SG, Chen JX, Li Q, Peng F, Zhu Z, Qin J and He ZY: The activation of ERK1/2 and JNK MAPK signaling by insulin/ IGF-1 is responsible for the development of colon cancer with type 2 diabetes mellitus. PLoS One 11: e0149822, 2016.

41. Araki W, Kume H, Oda A, Tamaoka A and Kametani F. IGFpromotes beta-amyloid production by a secretase-independent mechanism. Biochem Biophys Res Commun 380: 111-114, 2009

42. Zhou Q, Wang M, Du Y, Zhang W, Bai M, Zhang Z, Li Z and Miao J: Inhibition of c-Jun N-terminal kinase activation reverses alzheimer disease phenotypes in APPswe/PS1d E9 mice. Ann Neurol 77: 637-654, 2015.

43. Zhang Q, Li Y, Bao Y, Yin C, Xin X, Guo Y, Gao F, Huo S, Wang $X$ and Wang $Q$ : Pretreatment with nimodipine reduces incidence of POCD by decreasing calcineurin mediated hippocampal neuroapoptosis in aged rats. BMC Anesthesiol 18: 42, 2018.

44. Lin HH, Chen JH, Huang CC and Wang CJ: Apoptotic effect of 3,4-dihydroxybenzoic acid on human gastric carcinoma cells involving JNK/p38 MAPK signaling activation. Int J Cancer 120: 2306-2316, 2007

45. Scorrano L and Korsmeyer SJ: Mechanisms of cytochrome c release by proapoptotic BCL-2 family members. Biochem Biophys Res Commun 304: 437-444, 2003.

46. Kopeina GS, Prokhorova EA, Lavrik IN and Zhivotovsky B: Alterations in the nucleocytoplasmic transport in apoptosis: Caspases lead the way. Cell Prolif 51: e12467, 2018.

47. Shi Y: Mechanisms of caspase activation and inhibition during apoptosis. Mol Cell 9: 459-470, 2002.

48. Peña-Blanco A and García-Sáez AJ: Bax, Bak and beyond - mitochondrial performance in apoptosis. FEBS J 285: 416-431, 2018.

49. Festjens N, van Gurp M, van Loo G, Saelens $X$ and Vandenabeele P: Bcl-2 family members as sentinels of cellular integrity and role of mitochondrial intermembrane space proteins in apoptotic cell death. Acta Haematol 111: 7-27, 2004.

50. Yang L, Wang H, Liu L and Xie A: The role of insulin/IGF-1/ PI3K/Akt/GSK3 $\beta$ signaling in Parkinson's disease dementia. Front Neurosci 12: 73, 2018.

51. Ueki K, Fruman DA, Brachmann SM, Tseng YH, Cantley LC and Kahn CR: Molecular balance between the regulatory and catalytic subunits of phosphoinositide 3-kinase regulates cell signaling and survival. Mol Cell Biol 22: 965-977, 2002.

52. Dufour C, Holy X and Marie PJ: Transforming growth factorbeta prevents osteoblast apoptosis induced by skeletal unloading via PI3K/Akt, Bcl-2, and phospho-Bad signaling. Am J Physiol Endocrinol Metab 294: E794-E801, 2008.

53. Peruzzi F, Prisco M, Dews M, Salomoni P, Grassilli E, Romano G, Calabretta B and Baserga R: Multiple signaling pathways of the insulin-like growth factor 1receptor in protection from apoptosis. Mol Cell Biol 19: 7203-7215,1999.

54. WymannMP and PirolaL: Structure and function of phosphoinositide 3-kinases. Biochim Biophys Acta 1436: 127-150, 1998.

This work is licensed under a Creative Commons Attribution-NonCommercial-NoDerivatives 4.0 International (CC BY-NC-ND 4.0) License. 